\title{
Evaluation of Cortical Brain Parenchyma by Diffusion and Perfusion MRI Before and After Chronic Subdural Hematoma Surgery
}

\author{
Bekir AKGUN ${ }^{1}$, Hakan CAKIN², Sait OZTURK¹, Hanefi YILDIRIM ${ }^{3}$, Izzet OKCESIZ³, Saim KAZAN², \\ Fatih Serhat EROL ${ }^{1}$
}

${ }^{1}$ Firat University, Department of Neurosurgery, Elazig, Turkey

${ }^{2}$ Akdeniz University, Department of Neurosurgery, Antalya, Turkey

${ }^{3}$ Firat University, Department of Radiology, Elazig, Turkey

\section{ABSTRACT}

AIM: To evaluate microcirculatory changes in neighboring parenchyma as a result of pressure due to chronic subdural hematoma $(\mathrm{CSDH})$ in early and late periods after hematoma drainage.

MATERIAL and METHODS: The subject group consisted of 25 patients who underwent CSDH drainage. Brain diffusion and perfusion magnetic resonance images (MRIs) were obtained preoperatively, and at 48 hours (early period) and 2 months (late period) postoperatively. Measurements were performed on $1 \mathrm{~cm}^{2}$ regions of interest $(\mathrm{ROI})$ in the neighboring parenchymal tissue.

RESULTS: The early postoperative diffusion values showed improvement compared to the preoperative values. The late postoperative values showed improvement compared to the preoperative and early postoperative values. The early postoperative perfusion values showed slight decline compared to the preoperative values. However, the late postoperative values showed improvement compared to the preoperative and early postoperative values.

CONCLUSION: The fact that there was an increase in diffusion values from early to late postoperative periods, compared with the preoperative period, indicates that the beneficial effects of surgery increase over time. Brain perfusion was found to be slightly decreased in early postoperative period. Following CSDH drainage, neurological deteriorations are observed in some patients in the early postoperative periods; a slight impairment in perfusion may account for this. However, during the late postoperative period, perfusion was seen to recover prominently.

KEYWORDS: Chronic subdural hematoma, Diffusion, Perfusion, Magnetic resonance imaging

\section{INTRODUCTION}

$\mathrm{I}$ ntracranial subdural hematomas (SDHs) are hemorrhages that develop between the dura mater and the arachnoid mater and are likely to be associated with high mortality and morbidity. They are divided into three types as acute, sub-acute, and chronic, according to the time of development (7). Rupture of the parasagittal bridging veins, which course along the subdural distance, is classically shown as the factor responsible for the development of SDHs $(2,7)$. The causative factors for SDHs include previous trauma, coagulopathies, intracranial hypotension, chronic alcoholism, vascular malformations, and intracranial masses (1). According to the time of development, SDHs that develop within the first 3 days are classified as acute SDHs, those that develop within 4-20 days are classified as sub-acute SDHs, and those that develop after 21 days are classified as chronic $\mathrm{SDH}$ (CSDHs) (14). CSDHs reflect the organization of the 
blood that accumulates in the subdural distance. It is known that the amount of blood that accumulates in the subdural distance may gradually increase over time and may cause a mass effect. Pathophysiological mechanisms for this increase include osmotic-oncotic pressure differences, re-hemorrhage due to vulnerability of membrane capillaries, or fibrinolytic activation $(2,4,11)$.

Brain computed tomography (CT) and magnetic resonance imaging (MRI) are often used in the diagnosis of CSDHs. The structural characteristics of CSDHs can be examined using both CT and conventional MRI $(4,6,13,15)$. However, both of these imaging modalitities are inadequate in evaluating microcirculatory changes likely to occur due to chronic pressure on tissues in the vicinity of the hematoma. In contrast, diffusion and perfusion MRI sequences provide information not only on the structure of the space-occupying lesion, but also on the functional characteristics of the neighboring parenchymal tissue.

In this study, preoperative, early, and late postoperative brain diffusion and perfusion MRIs of patients who underwent CSDH surgical evacuation were evaluated. The main objective of this study was to identify early and late postoperative microcirculatory changes due to chronic pressure secondary to hematoma in the neighboring parenchymal tissue.

\section{MATERIAL and METHODS}

\section{Patients}

This study was conducted with Ethical approval (the approval date: September $5^{\text {th }}, 2013$ and number: 03/09) by the Ethics Committee of our university. The details of the study were explained either to the patients or to their legal representatives. After the informed consent forms were signed, the selected patients were incorporated into the study. Twenty five patients who underwent CSDH evacuation [age range: $42-89$ years, 7 females (28\%), 18 males (72\%)] were evaluated. Patients were included in the study if they had CSDH on first CT, a Glasgow Coma Scale score of 14 or 15 , and mild or no neurological deficit. Patients with moderate to severe neurological deficits and recurrent hemorrhage were excluded to avoid influencing the sensitivity of the statistical analysis. Brain diffusion and perfusion MRIs were taken a few hours preoperatively, and at 48 hours (early period) and 2 months (late period) postoperatively (Figures 1A-C; 2A-C).

\section{Radiological Examination}

The MRIs were taken using the General Electric 1.5 Tesla-brand MRI device with coils and 8-channel-neurovascular diffusion, perfusion, spectrography, and tractography features. The MRI images and the diffusion and perfusion measurement values were evaluated simultaneously by two radiologists and a neurosurgeon. Along with apparent diffusion coefficient (ADC) values obtained in $1 \mathrm{~cm}^{2}$ regions of interest (ROIs) neighboring the hematoma, diffusion measurements were performed. Regarding perfusion MRI evaluations, cerebral blood volume (CBV) measurements in the $1 \mathrm{~cm}^{2}$ ROls were performed with $\mathrm{ml} / 100-\mathrm{g}$ brain tissue units, and cerebral blood flow (CBF) measurements were performed with $\mathrm{ml} / 100 \mathrm{~g}$ brain tissue/ minute units.

\section{Surgical Interventions}

\section{Drainage with Burr Holes}

Patients with a hematoma thickness below $25 \mathrm{~mm}$, nonseptated hematoma content, and midline shift below 1 $\mathrm{cm}$, were operated via a burr hole technique under general anaesthesia.

On the side of the hematoma, two skin incisions of approximately $2 \mathrm{~cm}$ were made close to the anterior and posterior borders. Under these incisions, burr holes were opened into the calvarium at two locations using the Synthes Anspach Drill System. The hematoma in the subdural distance/space was drained. The involved area was washed with physiological saline solution. Irrigation was continued until fluid from the subdural distance became clear. The burr holes were closed using absorbable hemostatic gelatine sponges.

\section{Drainage via Craniotomy}

Patients with a hematoma thickness over $25 \mathrm{~mm}$, multipleseptated hematoma content, and midline shift over $1 \mathrm{~cm}$ underwent craniotomy under general anesthesia.

On the side of the hematoma, the skin was passed through with a horseshoe flap or a trauma flap bordering the thickest part of the hematoma, which was removed via craniotomy using the Synthes Anspach Drill System. The dura mater was incised and opened, and the hematoma was drained. The subdural space was washed with normothermic physiological saline solution. The membranes and septums within the subdural hematoma content were cleansed through careful dissection. The dura mater was then closed with primary suturing. The bone flap was replaced in its location and fixed with silk sutures.

\section{Statistical Analysis}

Statistical analyses were performed using the SPSS (Statistical Packet for the Social Science) 17.0 statistical package program. The Bonferroni-Dunn, Mann-Whitney $U$, and Z tests were performed. $p$ value $<0.05$ was accepted as statistically significant.

\section{RESULTS}

\section{Diffusion Measurement Values}

The mean diffusion MRI, ADC values were $0.822 \pm 0.06^{\prime} 10^{-3}$ $\mathrm{mm}^{2} / \mathrm{s}$ preoperatively, $0.864 \pm 0.06^{\prime} 10^{-3} \mathrm{~mm}^{2} / \mathrm{s} 2$ days postoperatively (early period), and $0.917 \pm 0.07^{\prime} 10^{-3} \mathrm{~mm}^{2} / \mathrm{s} 2$ months postoperatively (late period) (Figure 3). On pairwise comparison (Bonferroni test), a statistically significant difference was found between the preoperative and early postoperative period values $(p=0.003)$. There was also a statistically significant difference between the preoperative and late postoperative period values $(p<0.001)$. In addition, a statistically significant difference was found between the early and late postoperative period values $(p<0.001)$. The mean values showed an increase over time. 

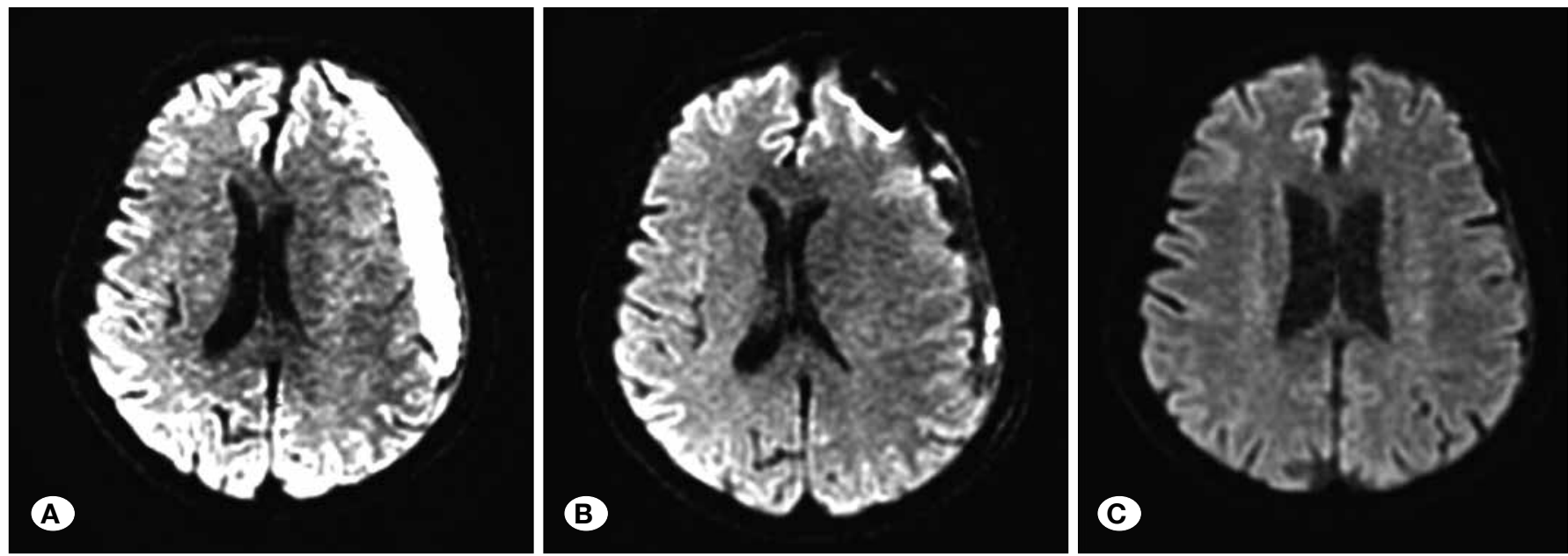

Figure 1: Diffusion weighted images of a 64-year old woman, who was included in this study. A) Preoperative, B) Postoperative $2^{\text {nd }}$ day, C) Postoperative $2^{\text {nd }}$ month.
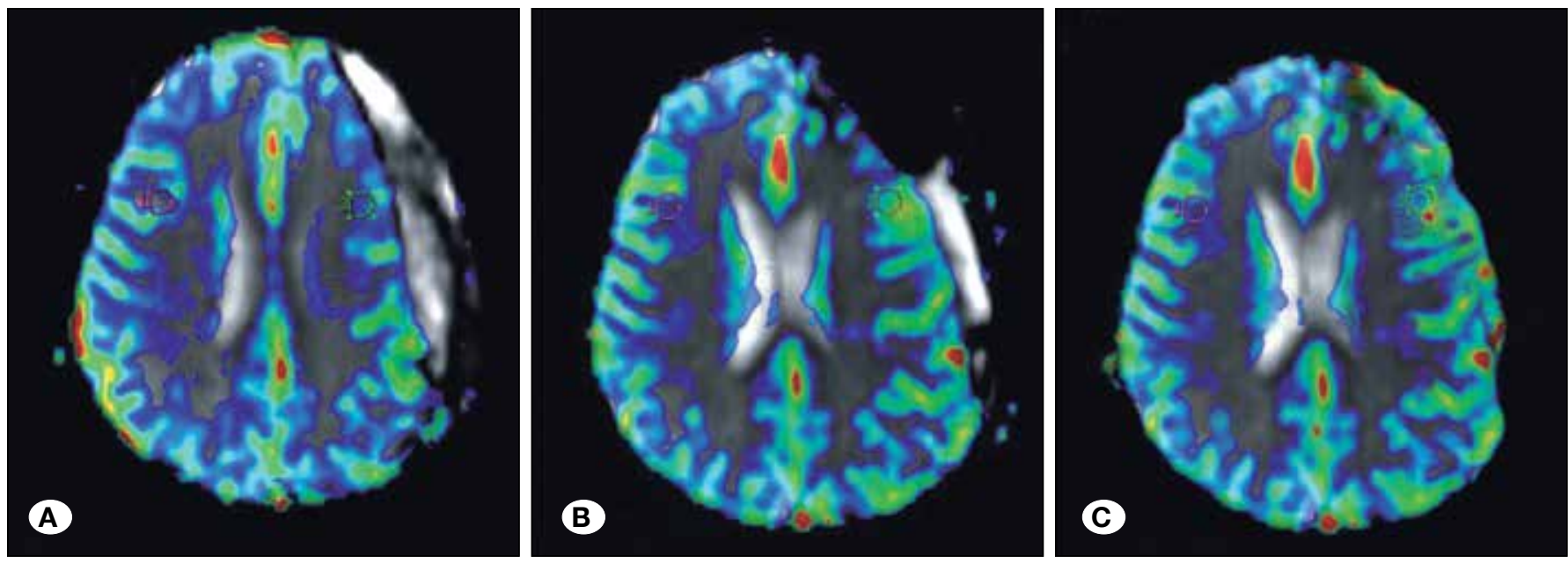

Figure 2: Perfusion MRI maps of a 64-year-old woman, who was included in this study. A) Preoperative, B) Postoperative $2^{\text {nd }}$ day, C) Postoperative $2^{\text {nd }}$ month (ROls; number 2 shows parenchyma next to the subdural hematoma).

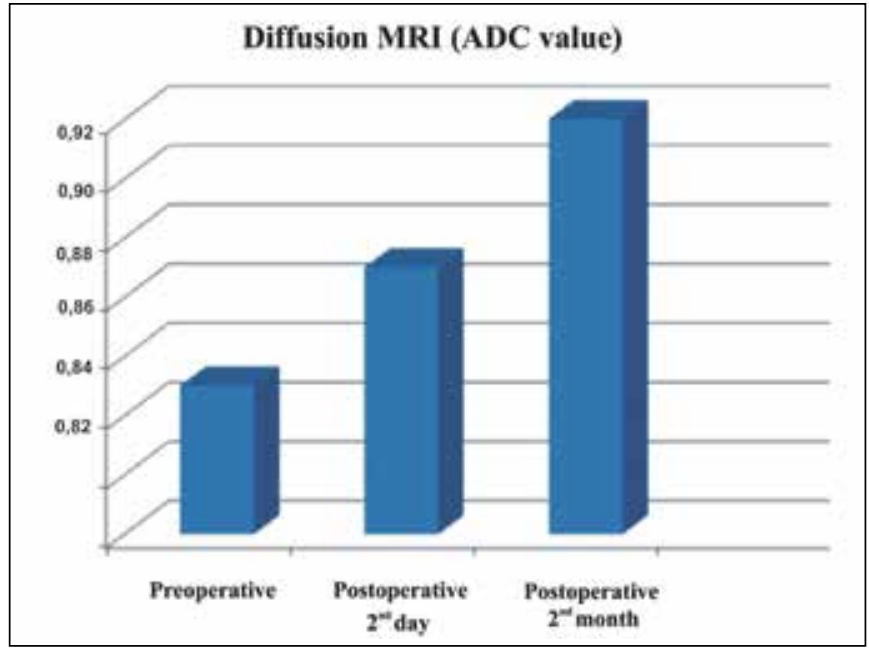

Figure 3: Diffusion measurement; mean ADC values (ADC: apparent diffusion coefficient).

\section{Perfusion Volume (Cerebral Blood Volume) Measurement Values}

The mean MRI perfusion volume measurement values were $2.72 \pm 0.5 \mathrm{ml} / 100 \mathrm{~g}$ brain tissue preoperatively, $2.63 \pm 0.4$ $\mathrm{ml} / 100 \mathrm{~g}$ brain tissue 2 days postoperatively (early period), and $3.13 \pm 0.7 \mathrm{ml} / 100 \mathrm{~g}$ brain tissue 2 months postoperatively (late period) (Figure 4). On pairwise comparison (Bonferroni test), there was a decline in the early postoperative period values compared to the preoperative values but it was statistically insignificant $(p=0.279)$.

On the other hand, a statistically significant difference was found between the preoperative and late postoperative period values $(p=0.021)$. In addition, a statistically significant difference was also found between the early and late postoperative period values $(p<0.001)$. The mean late postoperative period values were higher than those of the early preoperative periods. 


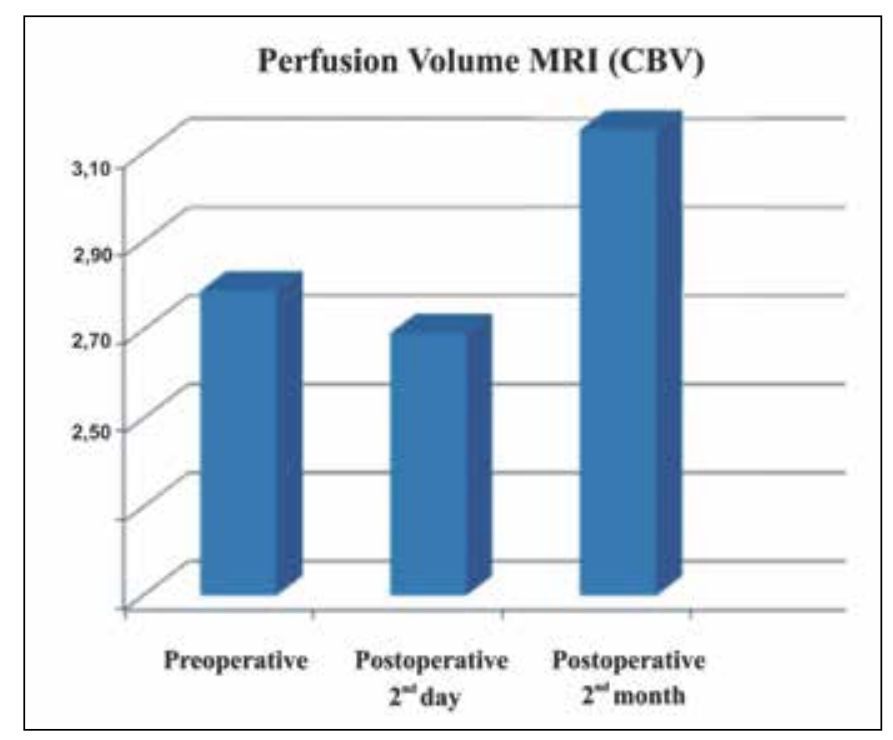

Figure 4: Mean perfusion volume MRI measurement values (CBV: cerebral blood volume).

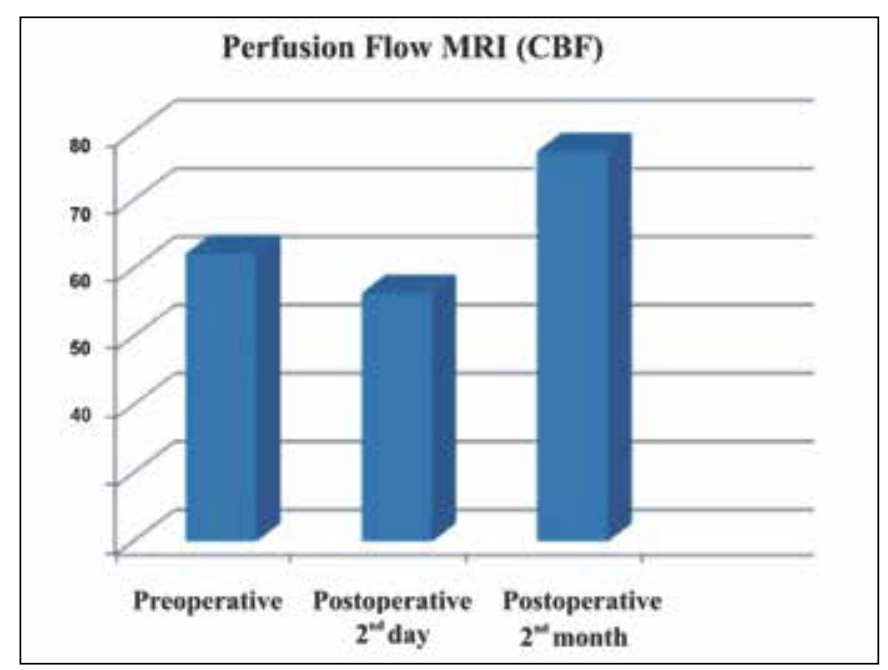

Figure 5: Mean perfusion flow MRI measurement values (CBF: cerebral blood flow).

\section{Perfusion Flow (Cerebral Blood Flow) Measurement Values}

The mean MRI perfusion flow measurement values were $60.1 \pm 3.2 \mathrm{ml} / 100 \mathrm{~g}$ brain tissue/min preoperatively, $54.8 \pm 2.4$ $\mathrm{ml} / 100 \mathrm{~g}$ brain tissue/min 2 days postoperatively (early period), and $75.7 \pm 3.4 \mathrm{ml} / 100 \mathrm{~g}$ brain tissue/min 2 months postoperatively (late period) (Figure 5). On pairwise comparison (Bonferroni test), there was a decline in the early postoperative period values compared to the preoperative values but it was statistically insignificant $(p=0.097)$. A statistically significant difference was found between the preoperative and late postoperative period values $(p=0.027)$. There was a statistically significant difference between the early and late postoperative period values $(p<0.001)$. The mean late postoperative period values were higher than those of the preoperative and early postoperative periods.

\section{DISCUSSION}

The pathophysiology of CSDH is not yet fully understood. The most commonly accepted theory is that the blood within the subdural space as a result of hemorrhage into the subdural region due to rupture or injury of bridging veins draining the cerebral surface toward the dural sinuses causes an inflammatory response $(2,7)$. Internal and external membranes are formed within the hematoma cavity. Fibroblasts entering the region and resultant granulation tissue are thought to be responsible for the membrane formation. Intermittent, new hemorrhages, coagulation, and increased fibrinolysis from new and fragile neovascularization, which is formed particularly over the external membrane, generate hematoma growth $(1,4,11)$. These mechanisms may be due to osmoticoncotic pressure differences, re-hemorrhage due to membrane capillary vulnerability, and fibrinolytic activation $(2,3,7,8)$. Hematomas, which become chronic depending on these mechanisms, are much thicker in the acute period and may cause midline shift. The idea behind this study was the existence of possible microcirculatory (diffusional and/ or perfusional) changes due to increasing and exacerbating chronic pressure in the neighboring parenchymal tissue as a result of gradual growth in hematoma size, because some CSDH patients complain of sudden neurological deficits, while others complain of gradually increasing deficits. Even when the hematoma is seen to be completely drained on postoperative radiological evaluation, temporary neurological regressions or fluctuations can sometimes be seen in the early postoperative period, which can last for a few days. For these reasons, the brain parenchyma next to the hematoma needs to be evaluated.

Due its variety of sequences, including diffusion, perfusion, and conventional imaging, as well as its high resolution, MRI is superior to CT for brain parenchymal tissue evaluation $(3,10,15)$. MRI consists of the transformation of different chemical and physical characteristics of hydrogen protons into images, which begin to resonate by interacting with the radiofrequency waves within a powerful magnetic field. Diffusion MRI is extremely sensitive to changes in the microscopic movements of water molecules and is used in clinical practice, particularly in the diagnosis of acute cerebral ischemia. ADC values represent the diffusion value of the water molecules within the tissue $(10,12)$. Cerebral perfusion MRI displays blood flow on a microscopic level. The degree of cerebral perfusion decrease correlates with changes that are likely to develop as a result of ischemic changes. Evaluation of cerebral perfusion can be performed using both volume and flow techniques. For cerebral perfusion volume, the amount of blood passing through $100 \mathrm{~g}$ brain tissue is measured (Cerebral blood volume). For cerebral perfusion flow, the amount of blood passing through $100 \mathrm{~g}$ brain tissue within a given period of time (1 minute) is measured (Cerebral blood flow) $(5,9)$.

Previous studies have examined subdural hematomas, their membranes, and internal structures using MRI $(4,6,13)$. However, to the best of our knowledge, no studies have examined microcirculatory changes in the neighboring 
parenchymal tissue by using diffusion and perfusion MRI together. In our study, we determined that diffusion in the brain parenchymal tissue neighboring the hematoma after $\mathrm{CSDH}$ drainage prominently recovered during both the early and late postoperative periods. Furthermore, diffusion in the late postoperative period was significantly increased compared to the early postoperative period, indicating the benefits of surgical treatment over time. However, compared to the preoperative period, we observed that perfusion slightly declined in the early postoperative period, whereas it prominently recovered in the late postoperative period.

There are two possible reasons as to why there is a decrease in brain perfusion in the early postoperative period even after hematoma drainage and release of parenchymal pressure. The first is that the tissue, the perfusion of which has already been disrupted by chronic pressure of the hematoma, is likely to be much more affected by anesthetic agents or hypotensive anesthesia. The second is that autoregulation, which regulates cerebral perfusion pressure, may reduce cerebral blood flow and volume through vasoconstriction in order to prevent reperfusion damage.

\section{- CONCLUSION}

The increase in diffusion of brain parenchymal tissue next to the hematoma after drainage, i.e., in the early and late postoperative periods compared to the preoperative period, suggests the benefits of surgical treatment over time. Even if the hematoma is successfully drained, there may still be clinical impairment in some patients during the early postoperative period. Our data suggest that a decrease in perfusion values might be responsible for these clinical impairments or fluctuations. However, brain perfusion prominently recovers in the late postoperative period.

\section{- REFERENCES}

1. Chen JC, Levy ML: Causes, epidemiology, and risk factors of chronic subdural hematoma. Neurosurgery Clin N Am 11: 399-406, 2000

2. Drapkin AJ: Chronic subdural hematoma: Pathophysiological basis of treatment. Br J Neurosurg 5: 467-473, 1991

3. Feng JF, Jilang JY, Bao YH, Liang YM, Pan YH: Traumatic subdural effusion evolves in to chronic subdural hematoma: Two stages of the same inflammatory reaction? Med Hypotheses 70: 1147-1149, 2008
4. Fujisawa H, Nomura S, Kajiwara K, Kato K, Fujii M, Suzuki $M$ : Various magnetic resonance imaging patterns of chronic subdural hematomas: Indicators of the pathogenesis? Neurol Med Chir (Tokyo) 46: 333-339, 2006

5. Kim H, Kim Y, Kim YW, Kim SR, Yang SH: Perfusion-weighted MRI parameters for prediction of early progressive infarction in middle cerebral artery occlusion. J Korean Neurosurg Soc 59: 346-351, 2016

6. Kuwahara S, Fukuoka M, Koan Y, Miyake H, Ono Y, Moriki A, Mori K, Mokudai T, Uchida Y: Subdural hyperintense band on diffusion-weighted imaging of chronic subdural hematoma indicates bleeding from outer membrane. Neurol Med Chir (Tokyo) 45: 125-131, 2005

7. Labadie EL, Glover D: Local alterations of hemostaticfibrinolytic mechanisms in reforming subdural hematomas. Neurology 25: 669-675, 1975

8. Markwalder TM: Chronic subdural hematomas: A review. J Neurosurg 54: 637-645, 1981

9. Rocchi G, Caroli E, Salvati M, Delfini R: Membranectomy in organized chronic subdural hematomas: Indications and technical notes. Surg Neurol 67: 374-380, 2007

10. Romero JM, Schaefer PW, Grant PE, Becerra L, Gonzalez RG: Diffusion MR imaging of acute ischemic stroke. Neuroimaging Clin N Am 12: 35-53, 2002

11. Senturk S, Guzel A, Bilici A, Takmaz I, Guzel E, Aluclu MU, Ceviz A: CT and MR imaging of chronic subdural haematomas: A comparative study. Swiss Med Wkly 140: 335-340, 2010

12. Shen $Q$, Duong TQ: Magnetic resonance imaging of cerebral blood flow in animal stroke models. Brain Circ 2: 20-27, 2016

13. Sundgren PC, Reinstrup P, Romner B, Holtas S, Maly P: Value of conventional, and diffusion-and perfusion weighted MRI in the management of patients with unclear cerebral pathology, admitted to the intensive care unit. Neuroradiology 44: 674680, 2002

14. Yeo CG, Jeon WY, Kim SH, Kim OL, Kim MS: The effectiveness of subdural drains using urokinase after burr hole evacuation of subacute subdural hematoma in elderly patients: A preliminary report. Korean J Neurotrauma 12: 101-106, 2016

15. Yokoyama K, Matsuki M, Shimano H, Sumioka S, Ikegana T, Hanabusa K, Yasuda S, Inoue H, Watanabe T, Miyashita M, Hiramatsu R, Murao K, Kondo A, Tanabe H, Kuroiwa T: Diffusion tensor imaging in chronic subdural hematoma: Correlation between clinical signs and fractional anisotropy in the pyramidal tract. Am J Neuroradiol 29: 1159-1163, 2008 